University of Nebraska - Lincoln

DigitalCommons@University of Nebraska - Lincoln

\title{
Time series analysis of jaw muscle contraction and tissue deformation during mastication in miniature pigs
}

\author{
Z. J. Liu \\ University of Washington \\ Jordan R. Green \\ University of Nebraska-Lincoln, jgreen4@unl.edu \\ C. A. Moore \\ University of Washington \\ S. W. Herring \\ University of Washington
}

Follow this and additional works at: https://digitalcommons.unl.edu/specedfacpub

Part of the Special Education and Teaching Commons

Liu, Z. J.; Green, Jordan R.; Moore, C. A.; and Herring, S. W., "Time series analysis of jaw muscle contraction and tissue deformation during mastication in miniature pigs" (2004). Special Education and Communication Disorders Faculty Publications. 52.

https://digitalcommons.unl.edu/specedfacpub/52

This Article is brought to you for free and open access by the Department of Special Education and Communication Disorders at DigitalCommons@University of Nebraska - Lincoln. It has been accepted for inclusion in Special Education and Communication Disorders Faculty Publications by an authorized administrator of DigitalCommons@University of Nebraska - Lincoln. 


\title{
Time series analysis of jaw muscle contraction and tissue deformation during mastication in miniature pigs
}

\author{
Z. J. Liu, ${ }^{1}$ J. R. Green, ${ }^{2}$ C. A. Moore, ${ }^{3}$ and S. W. Herring ${ }^{1}$ \\ 1. Department of Orthodontics, University of Washington, Seattle, WA, USA \\ 2. Department of Communicative Disorders, University of Wisconsin, WI, USA \\ 3. Department of Speech \& Hearing Science, University of Washington, Seattle, WA, USA \\ Corresponding author - Dr Z. J. Liu, Department of Orthodontics, School of Dentistry, University of Washington, \\ Box 357446, Seattle, WA 98195, USA; e-mail zjliu@u.washington.edu
}

\begin{abstract}
Masticatory muscle contraction causes both jaw movement and tissue deformation during function. Natural chewing data from 25 adult miniature pigs were studied by means of time series analysis. The data set included simultaneous recordings of electromyography (EMG) from bilateral masseter (MA), zygomaticomandibularis (ZM) and lateral pterygoid muscles, bone surface strains from the left squamosal bone (SQ), condylar neck (CD) and mandibular corpus (MD), and linear deformation of the capsule of the jaw joint measured bilaterally using differential variable reluctance transducers. Pairwise comparisons were examined by calculating the cross-correlation functions. Jaw-adductor muscle activity of MA and ZM was found to be highly cross-correlated with CD and SQ strains and weakly with MD strain. No muscle's activity was strongly linked to capsular deformation of the jaw joint, nor were bone strains and capsular deformation tightly linked. Homologous muscle pairs showed the greatest synchronization of signals, but the signals themselves were not significantly more correlated than those of non-homologous muscle pairs. These results suggested that bone strains and capsular deformation are driven by different mechanical regimes. Muscle contraction and ensuing reaction forces are probably responsible for bone strains, whereas capsular deformation is more likely a product of movement.
\end{abstract}

Keywords: electromyography, TMJ capsule, bone strain, mastication, jaw muscles, cross-correlation

\section{Introduction}

Muscle contractile forces are important for remodelling of soft and hard tissue. The loads generated on hard and soft tissue during muscle contraction are reported to be even greater than those from body weight (1). Such loads have two consequences for the tissues: stress and strain. As stress is not measurable in vivo, tissue deformation, or strain, assessed by in vivo strain gauge and differential variable reluctance transducer (DVRT) techniques, becomes a valuable indicator of the muscular loads (2). However, the spatial and temporal connectivity of muscle contraction and its mechanical effects on hard and soft tissue have not been well understood, because these bioelectrical signals have generally been treated as discrete events or single-point variables.

By using a variety of different time-dependent vari- ables such as electromyographic recordings (EMG), joint movement calculations, force measurements and bone surface strain gauge techniques, some researchers have applied correlation techniques to deduce functional connectivity or coordinative patterns of these parameters. The results of correlation analysis have shed light on how muscle contraction contributes to force production (3-7), and the coordination of motor behaviors $(8-16)$.

Mastication is a forceful cranial activity that produces obvious loads on the craniofacial components, especially on the jaw joint, the only diarthrosis (movable articulation) in the craniofacial complex. Nevertheless, the inherent correlations between the contractions of masticatory muscles and resulting loads or tissue deformations have not been explored as time-course variables, with the exception of Hylander and Johnson's reports $(3,4)$. 
They correlated masseter EMG with bone strain on the zygomatic arch during the power stroke of mastication to detect an optimal time constant for estimating the relative masseter force.

Cross-correlation analyses compare two given time series, or signals, at successive lags and provide two measurements. One is the maximum coefficient of the cross-correlation function, an index reflecting the relative similarity of the given two signals. The other is lag, an index of the synchrony of the two signals.

Our previous studies in pigs have demonstrated that the masseter muscle, the largest jaw adductor, is the major source of masticatory loads (17-19). The lateral pterygoid (LP) muscle is extremely important in protrusive movements but less important for loading (17). The zygomaticomandibular muscle is equivalent to the deep masseter in humans (20). In the present investigation, these three muscles were chosen as the targeted sources and their EMG activities during mastication were recorded. The deformations of both osseous and ligamentous tissues around the jaw joint were recorded simultaneously. Bone surface strains were measured by strain gauges and linear deformation of the lateral capsule of the jaw joint was measured by DVRT (Microstrain Inc., Burlington, VT, USA.). We hypothesized that: (i) activity of jaw adductors would be strongly coupled with bone strain and would precede bone strain; (ii) activity of LP muscle would be poorly coupled with bone strain, but would precede and be more strongly related to capsular deformation and (iii) similarly, bone and capsular deformations were not expected to be strongly coupled because of their respective association with loading and movement. Additionally, we investigated the coupling between homologous and non-homologous muscle pairs.

\section{Materials and methods}

\section{Data source}

No new experiments were performed in the present study. Instead, data were re-sampled from the previous chewing recordings on twenty-five 10-month-old Hanford miniature pigs (Sus scrofa, 12 males and 13 females) $(17,18)$. Thirteen of the pigs had received minor surgery to disrupt the lateral attachment of the capsule on the left jaw joint 5-6 weeks before the experimental observations. Prior to data collection, the animals were trained to feed in the recording apparatus. On the experimental day, pigs were anesthetized with halothane/nitrous oxide

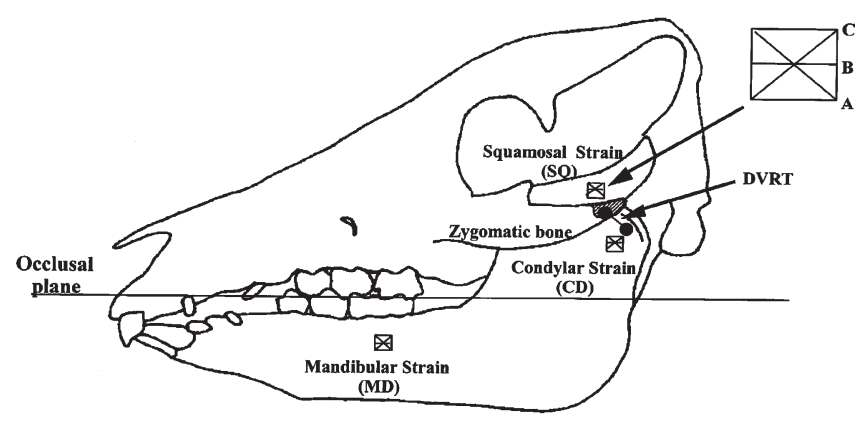

Figure 1. Left side of miniature pig skull showing the placement of 3 stacked rosette strain gauges on the lateral surface of the squamosal bone at the level of the articular eminence $(\mathrm{SQ})$, the lateral surface of the condylor neck (CD) and the lateral surface of the mandibular corpus (MD). Two solid dots and their connecting line indicate the implantation of the differential variable reluctance transducer (DVRT) in the lateral capsule of the jaw joint. The shaded area indicates the caudal tip of the zygomataic bone, which was removed to facilitate DVRT implantation. A, B, and C in the enlargement represent the 3 elements of each gauge.

for surgical placement of strain gauges, DVRTs and EMG electrodes. Stacked 3-element rosette strain gauges (SK06-030WR-120; Measurement Groups Inc., Raleigh, NC, USA) were bonded to the lateral surface of the left squamosal bone at a location in line with the articular eminence (SQ), the lateral surface of left condylar neck (CD), and the lateral aspect of the left mandibular corpus below the first molariform teeth (MD) with cyanoacrylate glue. Prior to placement of the strain gauges, each bone site was exposed and prepared through cauterization, smoothing, neutralizing and drying. The rosette strain gauge measures simultaneous strain in three directions ( $45^{\circ}$ separated from each other) and allows the calculation of the magnitudes and directions of principal (compressive and tensile) strains (21). The DVRT was secured by inserting its barbs into the lateral ligament of each jaw joint capsule (L/R DVRT) with its long axis oriented from anterosuperiorly to posteroinferiorly. The DVRT is an arthroscopically implantable transducer suitable for measuring the linear deformation of soft tissue, especially ligamentous components of joints (22) and allows the calculation of elongation or shortening and strain (displacement divided by the original length) of the soft tissue during function or manipulation. Figure 1 illustrates the placements of three rosette strain gauges and one DVRT on the left side. After strain gauge and DVRT placement, fine-wire electrodes (0.05-mm nickel-chromium wire, $1 \mathrm{~mm}$ bared tip) were inserted percutaneously into the bi- 

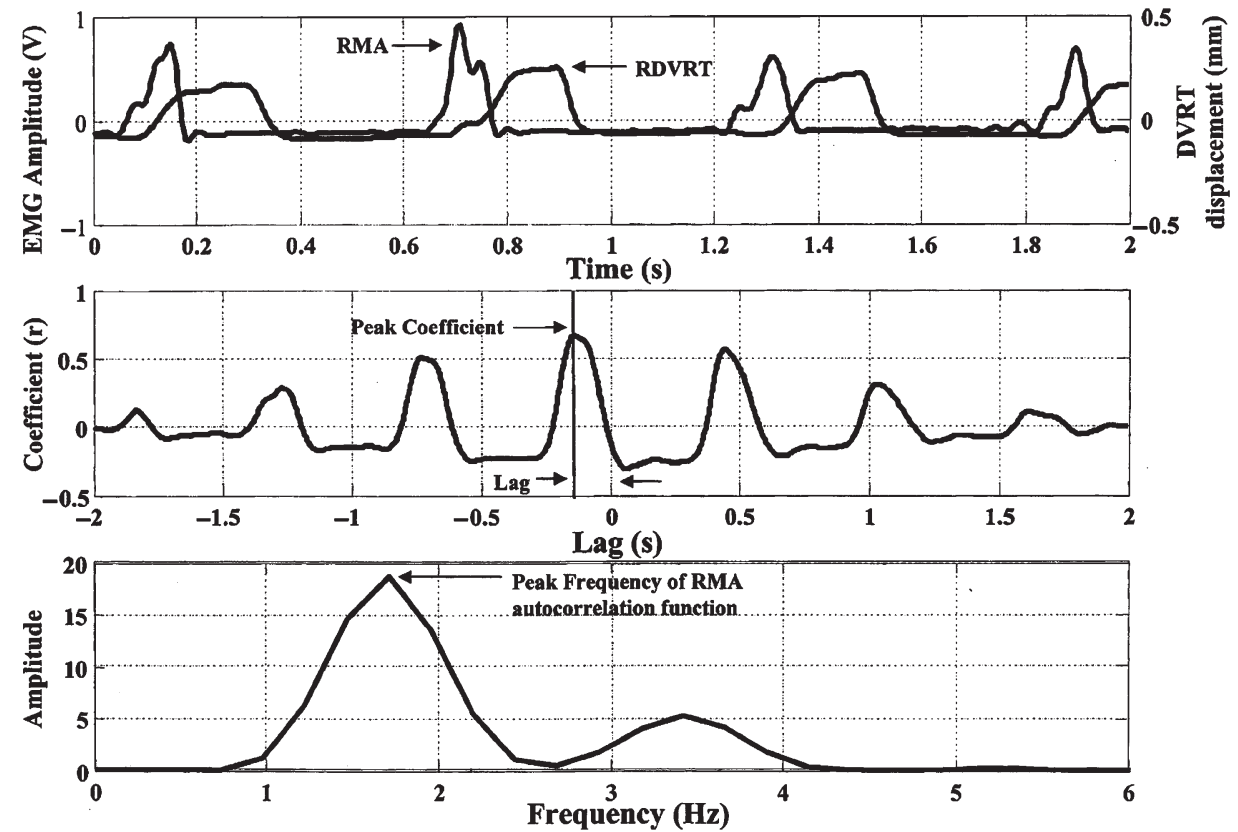

Figure 2. Two-second recording, showing full-wave rectified and smoothed (3 points) EMGs (masseter, MA, zygomaticomandibularis, ZM, and lateral pterygoid, LP), squamosal (SQ), condylar (CD) and mandibular (MD) bone strains (only one of three elements in each location is shown) and left capsular deformation (DVRT). The dashed vertical lines indicate the rough starting points of each chewing cycle, as indicated by the onset of masseter activity. For the strain gauge signals, upward and downward deflections over baseline represent tensile and compressive strains, respectively. For the DVRT signal, upward and downward deflections correspond to elongation and shortening in the lateral capsule of the jaw joint, respectively.

lateral masseter (MA), zygomaticomandibular (ZM) and LP muscles. The accuracy of electrode position was verified by back stimulation. Further details about installation, calibration and recording equipment are described elsewhere $(17,18)$. Local anesthetic $(2 \%$ procaine hydrochloride) was drizzled onto the incisions and an analgesic (buprenorphrine hydrochloride) was administered intramuscularly. After recovery from anaesthetic, the unrestrained animal was fed pig chow pellets, and cookies or biscuits were offered in a few cases as well. Two pigs refused food but chewed when a piece of rubber or a tongue depressor was placed between the teeth. While pigs were chewing, the amplified signals of EMG, bone strain and DVRT displacement were collected at $500 \mathrm{~Hz}$ and stored for offline analysis (MP100; Biopac Systems, Inc., Goleta, CA, USA). Signals were recorded continuously for 20$30 \mathrm{~min}$, and stored in files that contained about $3 \mathrm{~min}$ of data. Figure 2 illustrates the rectified EMGs, bone strains, and DVRT displacement. The DVRT and gauge sites were checked post-mortem, and data were discarded if devices were improperly placed. The successful sample sizes from each source are listed in Table 1. The animal use protocol was reviewed and approved by the University of Washington Institutional Animal Care and Use Committee.
Table 1. Sample size for each transducer and electrode

\begin{tabular}{lcc}
\hline & \multicolumn{2}{c}{ Number of subjects } \\
\cline { 2 - 3 } & Left & Right \\
\hline Strain gauge & 22 & \\
$\quad$ Squamosal site (SQ) & 18 & \\
$\quad$ Condylar site (CD) & 10 & 9 \\
$\quad$ Mandibular site (MD) & 15 & 15 \\
DVRT & 15 & 5 \\
EMG & 4 & 9 \\
$\quad$ Masseter (MA) & 13 & \\
Zygomaticomandibularis (ZM) & & \\
$\quad$ Lateral pterygoid (LP) & & \\
DVRT, differential variable reluctance transducer; EMG, \\
electromyogram.
\end{tabular}

\section{Data processing}

For each pig, the best stereotypical chewing sequence was selected based on EMG signal regularity [consistent in amplitude, without interruption, and showing alternation of chewing side; (23), also refer to Figure 2]. Two 
Figure 3. Upper: the conditioned signals from RMA (solid curve) and RDVRT (dotted curve) during 2seconds of chewing collected from a single subject. Midddle: the results of the cross-correlation analysis of the RMA and RDVRT signals displayed in the upper panel. Similarity and synchrony between two signals were determined by extracting the peak coefficient and associated lag from the cross-correlation function, respectively. Coefficient $(\mathrm{r})=$ 0.64, Lag $=125$ ms. Bottom: chewing frequency (about $1.7 \mathrm{~Hz}$ ) was calculated by identifying the dominant peak in the spectrum of the auto-correlation of the conditioned RMA signal.

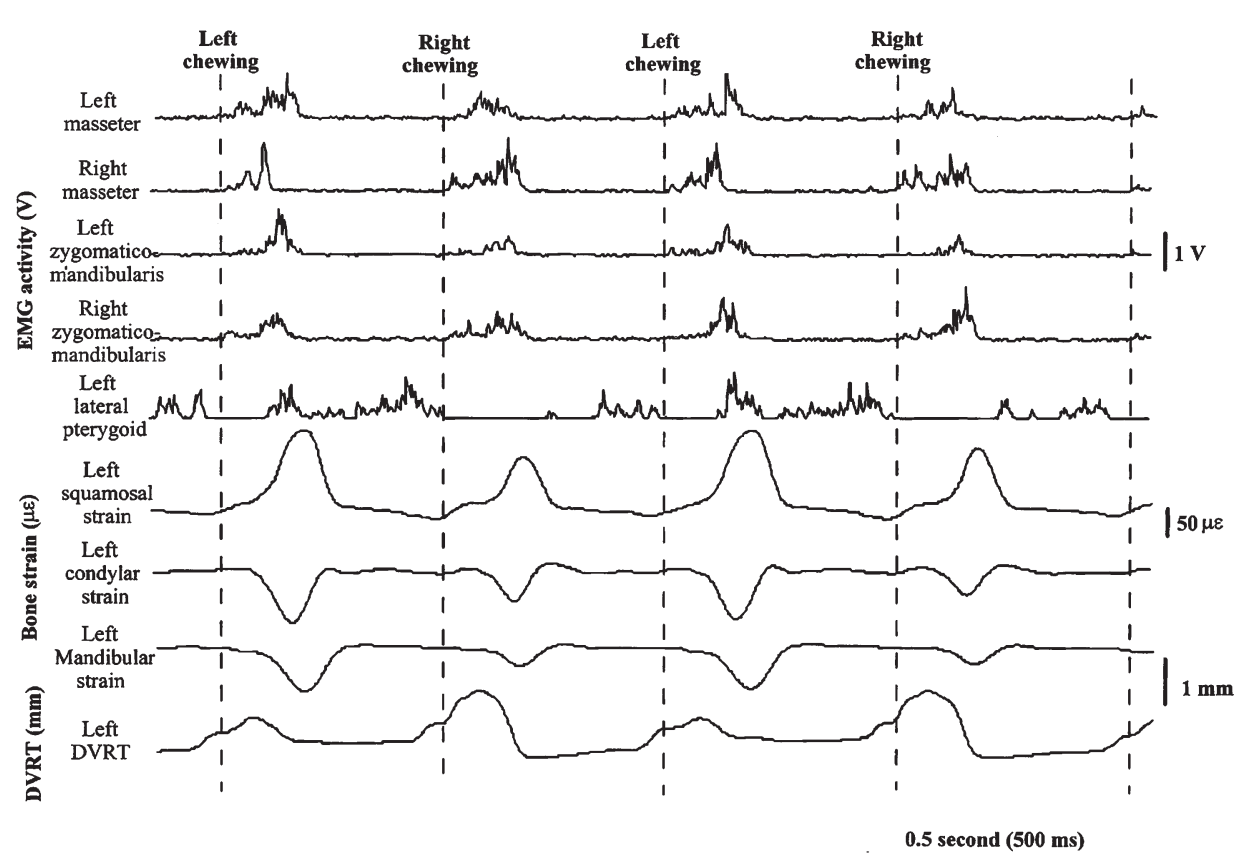

seconds of data representing approximately four chewing cycles were extracted from each selected chewing episode. Preprocessing of the EMG data involved fullwave rectification followed by zero-phase digital filtering (Butterworth Filter, low-pass cutoff $=30 \mathrm{~Hz}$; Biopac Systems, Inc., Goleta, CA, USA). The digitized EMG and DVRT waves were not manipulated further. However, the three signals (channels A, B, C, Figs 1 and 2) from each strain gauge were transformed into a single vector that represented shear strain. The formulae used to transform these data were provided by the manufacturer (24), and involved summing the absolute value of principal compressive and tensile strains. This value was taken to represent the corresponding bone strain.

Cross-correlation analysis was applied to the extracted and treated signals using algorithms custom designed for MATLAB (Ver. 4.2c; The MathWorks Inc., Natick, MA, USA), a commercially available signalprocessing package. Each signal was centered about its mean prior to correlational analysis. Cross-correlation functions, including the auto-correlation function for the left MA signal (right MA, if left was unavailable), were computed for 2-s intervals in a pairwise manner for the data obtained from each pig. Figure 3 illustrates the derivation of the two indices used to quantify the strength and timing of the time domain signals studied, and includes two-second samples of one pair of signals used in these computations. The upper panel includes the smoothed and rectified MA EMG (solid line) and the
DVRT displacement of the right lateral capsule (dotted line). It is evident in this panel that RMA activity predicts increases in the RDVRT, and further, that RMA activity precedes increases in RDVRT. The strength of this predictability and the asynchrony of the cross-correlated signals are quantified by the peak cross-correlation coefficient and the lag associated with that peak, respectively. These two values were derived from the crosscorrelation function obtained for each pair. The middle panel of Figure 3 illustrates this function for the signals shown. The peak coefficient $(r=0.64)$ was observed at a lag of $-125 \mathrm{~ms}$, indicating a relatively high degree of predictability between the two signals, with the RMA signal leading the RDVRT signal by $125 \mathrm{~ms}$. A further aspect of this analysis was the computation of the autocorrelation function of each signal, the peak of which indicates the dominant period of the signal (13). The spectrum of the autocorrelation function obtained for the RMA EMG in the upper panel is shown in the lower panel of Figure 3. The periodicity, which can be seen in the upper panel to be approximately $600 \mathrm{~ms}$, yielded a peak in the spectrum of the autocorrelation function (lower panel) of $1.7 \mathrm{~Hz}$.

\section{Signal pairs}

Preliminary testing (two-sample t-test on both peak coefficient and lag time) indicated that there were no significant right-left differences. This finding was ex- 
pected because the alternating chewing pattern of pigs results in both left- and right-side chewing cycles being included in every 2-s data set. Therefore, muscle and capsular data from both sides were combined for comparison of bone strains. Data from the surgery and non-surgery groups were originally kept separate. However, preliminary testing again demonstrated that there were no significant differences. This result was also expected, based on our previous findings that the minor surgery on the lateral capsule of the jaw joint had no substantial effect on chewing performance (17), jaw movement $(25)$ or functional loading $(17,18)$. Hence, the data from both groups were combined. The following categories were examined: (i) three muscles (MA, ZM and LP) with three bone surface strains (left $\mathrm{SQ}, \mathrm{CD}$ and $\mathrm{MD}$ ). This category yielded eight (no data were available for the pair of $\mathrm{ZM}$ with $\mathrm{MD}$ ) pairwise cross-correlation functions; (ii) capsular deformation (two DVRTs) with two muscles (MA and LP, unavailable data for ZM) and three bone strains (left SQ, CD and MD). This category yielded five pairwise crosscorrelation functions; (iii) three bone strains paired with each other and two DVRTs (left and right capsules of jaw joint) paired with each other. This category yield four pairwise cross-correlation functions and (iv) pairs of homologous (left and right MA, ZM and LP) and non-homologous muscles (MA with $\mathrm{ZM}$ and with LP, insufficient data for comparison of ZM with LP). This category yielded five pairwise cross-correlation functions.

\section{Statistical analysis}

Analysis of variance (one-way ANOVA) and post hoc Bonferroni (for muscle-strain pairs) or Tukey tests were performed to detect differences in multiple pairwise comparisons. The t-tests were carried out for two-sample comparisons. The lags were converted to absolute values before the above statistical comparisons to minimize the effect of the order of signal development. The lag's sign was only used to detect the temporal order of the compared signals' predicted variability. For example, a positive sign of a muscle-strain pair indicates that the muscle activity best predicted bone strain signal that occurred later in the record, and vice versa. To examine the differences of the lag signs, Chi-square tests were carried out for multiple comparisons, while Kruskal-Wallis H or Mann-Whitney U tests were performed to detect sign differences between pairs. Probability levels of 0.05 or less were considered to indicate statistical significance.

\section{Results}

\section{Chewing frequency}

The autocorrelation functions calculated for the masseter showed that chewing frequency ranged from 1.46 to $2.20 \mathrm{~Hz}$ with a mean of $1.87 \mathrm{~Hz}$ (s.d. $=0.24$ ), i.e. about $535 \mathrm{~ms}$ per chewing cycle.

\section{Pairs of muscle EMGs with bone strains}

The activities of MA and especially ZM were strongly coupled with the SQ and CD strains as indicated by cross-correlation peak coefficients ranging from 0.61 to 0.78 . The weakest coupling was between MA and MD strain $(r=0.441)$. The difference between $\mathrm{ZM}-\mathrm{CD}$ and MA-MD was highly significant $(P=0.008)$. The LP was moderately correlated with bone strain in all three locations with peak coefficients ranging from 0.53 to 0.58 (Table 2).

Muscle-strain pairs varied greatly in lag times. The MA and ZM were well synchronized with strains in SQ and CD locations (49-79 ms, $<15 \%$ of chewing cycle length). The LP was significantly less synchronous with SQ and CD strains, with lags of $145-152 \mathrm{~ms}(>25 \%$ of chewing cycle length, $\mathrm{P}=0.007-0.038$ ). Interestingly, MA and LP were both synchronized fairly well with MD strain (79 and $85 \mathrm{~ms}$, respectively).

The signs of the lags revealed that MA activity significantly preceded SQ and CD strains $(82-92 \%, P<0.001$ and $=0.003$, respectively); $\mathrm{ZM}$ showed a similar trend (78-86\%, P $=0.059$ and 0.096, respectively). However, MA did not consistently precede MD strain (44\%), and LP did not have a consistent order with any strain signal (50-56\%).

\section{Pairs of capsular deformations (DVRT) with muscle EMGs and bone strains}

No significant main effect for peak coefficient was found among DVRT-muscle pairs. These coefficients $(r=0.39-0.52)$ were generally weaker than those of muscle EMGs with bone strains (Table 3).

The lag values indicated that capsular deformations were asynchronous with muscle EMGs or bone strains (all $>100 \mathrm{~ms}$, about $20 \%$ of the chewing cycle length). Values were similar to those of the LP with SQ and CD strains (compare Tables 2 and 3).

Statistical analysis revealed that capsular deformation usually preceded MA activity (75\% negative lag; $\mathrm{P}=0.014)$, but showed no significant relationship to LP 


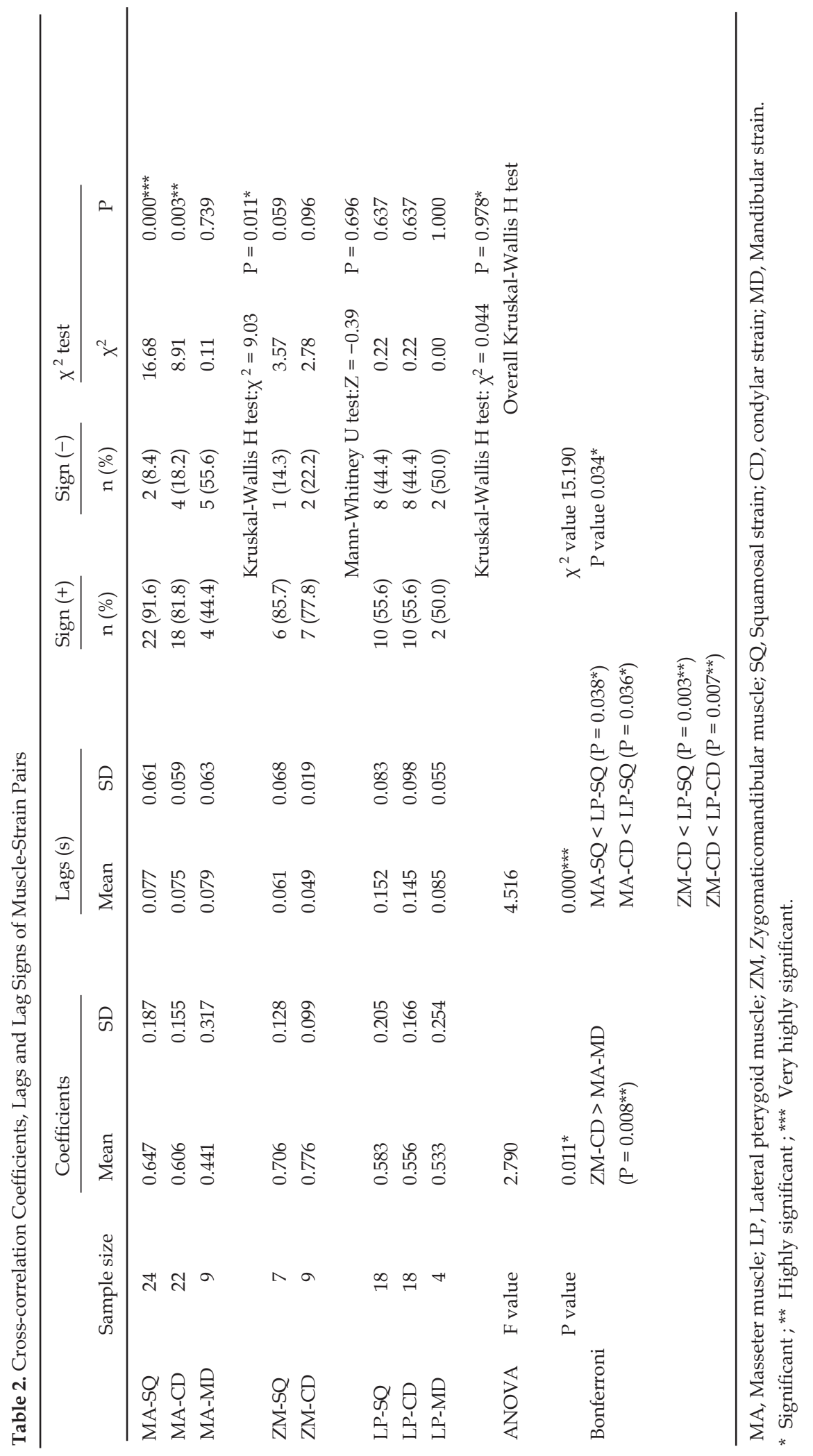


Table 3. Cross-correlation Coefficients, Lags and Lag Signs of DVRT-Muscle and DVRT-Bone Strain Pairs

\begin{tabular}{|c|c|c|c|c|c|c|c|c|c|}
\hline & \multirow[b]{2}{*}{ Sample size } & \multicolumn{2}{|c|}{ Coefficients } & \multicolumn{2}{|c|}{ Lags (s) } & \multirow{2}{*}{$\frac{\operatorname{Sign}(+)}{\mathrm{n}(\%)}$} & \multirow{2}{*}{$\frac{\operatorname{Sign}(-)}{\mathrm{n}(\%)}$} & \multicolumn{2}{|c|}{$x^{2}$ test } \\
\hline & & Mean & SD & Mean & $\mathrm{SD}$ & & & $x^{2}$ & $\mathrm{P}$ \\
\hline DVRT-MA & 24 & 0.516 & 0.197 & 0.131 & 0.069 & $18(75.0)$ & $6(25.0)$ & 6.00 & $0.014^{*}$ \\
\hline DVRT-LP & 13 & 0.493 & 0.170 & 0.129 & 0.073 & $4(38.8)$ & $9(69.2)$ & 2.57 & 0.109 \\
\hline t-test & $t$ value & 0.370 & & 0.138 & & \multicolumn{2}{|c|}{ Mann-Whitney U test } & \multicolumn{2}{|c|}{$\mathrm{Z}$ value -2.76} \\
\hline DVRT-CD & 16 & 0.416 & 0.191 & 0.128 & 0.084 & 15 (93.7) & $1(6.3)$ & 12.25 & $0.000^{* * *}$ \\
\hline DVRT-MD & 9 & 0.389 & 0.218 & 0.137 & 0.099 & $8(88.9)$ & 1 (11.1) & 5.44 & $0.020^{*}$ \\
\hline \multirow[t]{2}{*}{ ANOVA } & F value & 1.532 & & 0.503 & & \multirow{2}{*}{\multicolumn{2}{|c|}{ Kruskal-Wallis $\mathrm{H}$ test }} & \multicolumn{2}{|c|}{$x^{2}$ value 5.36} \\
\hline & P value & 0.229 & & 0.608 & & & & \multicolumn{2}{|c|}{ P value 0.069} \\
\hline
\end{tabular}

DVRT, Differential variable reluctance transducer; MA, Masseter muscle; LP, Lateral pterygoid muscle; SQ, Squamosal strain; CD, condylar strain; MD, Mandibular strain.

* Significant ; ** Highly significant ; ${ }^{* * *}$ Very highly significant

activity $(69 \%$ positive lag; $\mathrm{P}=0.11)$. A Mann-Whitney $\mathrm{U}$ test indicated a significant difference between these two pairs $(\mathrm{P}=0.006)$. Capsular deformation preceded bone strains (63-94\%), showing a significantly greater proportion of observations for the CD and MD locations (88$93.7 \%, \mathrm{P}<0.001$ and $=0.02$, respectively), but not for the SQ location (63\%; $\mathrm{P}=0.32)$.
Pairs of bone strains and paired capsular deformations

The CD-SQ strain pair showed significantly higher coupling $(r=0.73)$ than the CD-MD pair $(r=0.48$, $\mathrm{P}=0.016)$, and was higher than the SQ-MD pair $(\mathrm{r}=0.43, \mathrm{P}=0.053)$. These findings reinforce the similarity of the time course of SQ and CD strain and their difference from MD strain. Left and right capsular deformations were moderately similar with a peak coefficient of 0.61 (Table 4 ).

Table 4. Cross-correlation Coefficients, Lags and Lag Signs of Strain Pairs and Capsular Pair

\begin{tabular}{|c|c|c|c|c|c|c|c|c|c|}
\hline & Sample size & \multicolumn{2}{|c|}{ Coefficients } & \multicolumn{2}{|c|}{ Lags (s) } & $\frac{\operatorname{Sign}(+)}{\mathrm{n}(\%)}$ & $\frac{\operatorname{Sign}(-)}{\mathrm{n}(\%)}$ & \multicolumn{2}{|c|}{$x^{2}$ test } \\
\hline \multicolumn{10}{|c|}{ Strain Pairs } \\
\hline SQ-MD & 6 & 0.482 & 0.273 & 0.098 & 0.101 & $6(100.0)$ & $0(0.0)$ & - & $0.000^{* * *}$ \\
\hline CD-MD & 8 & 0.425 & 0.337 & 0.043 & 0.048 & $8(100.0)$ & $0(0.0)$ & - & $0.000^{* * *}$ \\
\hline ANOVA & P value & $0.011^{*}$ & & 0.432 & & \multicolumn{2}{|c|}{ Kruskal-Wallis H test } & $\mathrm{p}$ value 0 & \\
\hline \multirow[t]{2}{*}{ Tukey test } & \multicolumn{9}{|c|}{ CD-SQ > SQ-MD $\left(P=0.016^{*}\right)$} \\
\hline & \multicolumn{9}{|c|}{ CD-SQ $>$ CD-MD $(P=0.053)$} \\
\hline \multicolumn{10}{|c|}{ Capsular pair } \\
\hline
\end{tabular}

SQ, Squamosal strain; CD, condylar strain; MD, Mandibular strain; DVRT, Differential variable reluctance transducer.

* Significant; *** Very highly significant 
The lags of bone strain pairs exhibited great variation (CV > 100\%). Although this variability mitigated against detecting significant differences, it is notable that the CD-MD pair exhibited the shortest lag $(43 \mathrm{~ms})$ of all comparisons. These locations are on the same bone, the mandible. Left and right capsular deformation had a relatively long, highly variable lag (109 ms, CV = 102\%).

The lag signs further demonstrated that $\mathrm{CD}$ strain developed prior to the SQ strain $(75 \%, \mathrm{P}=0.046)$, while MD strain was always last $(100 \%, \mathrm{P}<0.001)$, resulting in a significant difference among these three pairs $(\mathrm{P}<0.001)$.

\section{Muscle pairs}

Comparison of pairs of homologous muscles revealed no significant differences, with coefficients ranging from 0.72 for $\mathrm{L} / \mathrm{R} \mathrm{ZM}$, to 0.56 for $\mathrm{L} / \mathrm{R} \mathrm{MA}$ and 0.53 for $\mathrm{L} / \mathrm{R}$ LP. The synergistic non-homologous muscle pair (MA$\mathrm{ZM}, \mathrm{r}=0.75$ ) exhibited significantly stronger coupling than the antagonistic muscle pair (MA-LP, $r=0.56$, $\mathrm{P}<0.001$ ), and this coupling was significantly stronger than for the homologous muscle pairs of MA and LP ( $t$ test, $\mathrm{P}=0.023$ and 0.041 , respectively) (Table 5).

The lags of the three pairs of homologous muscles were the shortest ones observed (11-13 ms, significantly shorter than all comparisons, $\mathrm{P}<0.05,0.01$, or 0.001$)$, whereas they did not differ from each other. Thus, homologous muscles were activated in a synchronous mode during mastication, and side differences in timing were generally $<3 \%$ of the chewing cycle length. Consistent with the peak coefficients, the synergistic muscle pair (MA-ZM) showed significantly better synchrony than the antagonistic muscle pair (MA-LP, $\mathrm{P}<0.001$ ).

When the lags' signs were checked to identify the order of activation, it was found that the activity of MA significantly preceded that of LP $(88 \%, \mathrm{P}=0.001)$. The order for the MA-ZM pair was inconsistent.

\section{Discussion}

\section{Cross-correlation analysis}

Traditional approaches describing wave signal patterns have relied on the identification of single points (for example, onset or offset of EMG burst) or use discrete events (for example, stimulation trigger). Crosscorrelation analysis provides a method by which asynchronous signals can be evaluated with respect to their similarity and temporal offset. Thus, this method overcomes some disadvantages of traditional techniques, such as experimenter bias and measurement error. Cross-correlation analysis has been frequently used to deduce functional connectivity within neuronal circuits and between neurons and muscles (Schwartz \& Adams, 1995) and is an effective way to detect common periodicities in two signals of interest (15). The other primary

Table 5. Cross-correlation Coefficients, Lags and Lag Signs of Muscle Pairs

\begin{tabular}{|c|c|c|c|c|c|c|c|c|c|}
\hline & Sample size & \multicolumn{2}{|c|}{ Coefficients } & \multicolumn{2}{|l|}{ Lags (s) } & $\frac{\operatorname{Sign}(+)}{\mathrm{n}(\%)}$ & $\frac{\operatorname{Sign}(-)}{\mathrm{n}(\%)}$ & \multicolumn{2}{|c|}{$x^{2}$ test } \\
\hline \multicolumn{10}{|c|}{ Homologous pairs } \\
\hline $\mathrm{L} / \mathrm{R} \mathrm{ZM}$ & 4 & 0.718 & 0.097 & 0.012 & 0.016 & - & - & - & - \\
\hline L/R LP & 4 & 0.530 & 0.124 & 0.013 & 0.010 & - & - & - & - \\
\hline ANOVA & $\mathrm{P}$ value & 0.202 * & 0.992 & & & & & & \\
\hline \multicolumn{10}{|c|}{ Non-homologous pairs } \\
\hline MA-ZM & 10 & 0.747 & 0.123 & 0.022 & 0.020 & $7(70.0)$ & $3(30.0)$ & 1.60 & 0.317 \\
\hline MA-LP & 25 & 0.558 & 0.125 & 0.186 & 0.040 & $21(87.5)$ & $4(22.5)$ & 11.56 & $0.001^{* *}$ \\
\hline
\end{tabular}

L/R, Left vs. Right; MA, Masseter muscle; ZM, Zygomaticomandibular muscle; LP, Lateral pterygoid muscle.

* Significant ; ** Highly significant ; *** Very highly significant 
advantage of this method is that it permits reduction of very large samples to comprehensible and statistically testable values (12).

The magnitude and intensity of the EMG signal is at least qualitatively related to the force produced by a muscle under certain conditions (7). However, the relationship of muscle activity to tissue deformation has not been well quantified. The difficulties of relating EMG to the corresponding tissue deformation are associated with the delay between muscle activation and mechanical responses, the difficulty in reliably measuring EMG associated with dynamic movement, and the presence of many tissues with varying non-linear mechanical properties. The present study attempted to overcome these difficulties by a comprehensive timeseries analysis of the coupling between masticatory muscle contractions and tissue deformations around the jaw joint. Although the results were encouraging and mostly explainable, there are a number of methodological factors that require further attention. First, the methods used in the present study could not ensure that each data set was evenly distributed between left and right chewing cycles. This imbalance would yield increased variability because working and balancing sides have different EMG and DVRT patterns (17). Secondly, time-series analysis gives the clearest results for periodic or stereotyped signals. However, during free mastication, some signals are complex and variable. In particular, the LP muscle often has two separate bursts of activity during a chewing cycle (17), and the bone strain on CD may also show a 2-phase pattern (2). Depending on the individual cycle or pig, the cross-correlation computation may identify a different phase as the peak. This inconsistency contributes to the variance observed.

\section{Jaw muscle contractions and bone deformations}

Studies of in vivo strain in the zygomatic arch have indicated that the most likely cause of bending of the SQ during mastication is the inward pull of the MA and the ZM $(17-19,26)$. Condylar strain, however, results from the reaction force, a downward force exerted by the articular eminence onto the condyle when the upward-acting jaw adductors contract (2). Thus, strong couplings between the MA/ZM muscles and the SQ/CD strains are expected. In the present study, the coupling between two jaw adductors (MA and $\mathrm{ZM}$ ) and two bone strains (SQ and $\mathrm{CD}$ ) were strong, even greater than those of homologous MA and LP pairs (compare Tables 2 and 5). These results provide strong support for the previous suggestions that these two muscles have a direct relationship with these two strains.

Further, the lags and their signs suggest that this relationship is causal. Hylander and Johnson $(3,4)$ estimated that surface masseter EMG precedes muscle force by 22$72 \mathrm{~ms}$ during the power stroke of mastication. Consistent with this estimate, the current study found a lag of about 75 ms between the MA/ZM EMGs and the SQ/ $\mathrm{CD}$ bone strains, with muscle activity usually preceding the bone strains. Moreover, these lag values were significantly longer ( $>10 \%$ of chewing cycle) than those of homologous and synergistic MA-ZM muscle pairs $(<5 \%$ of chewing cycle length, $\mathrm{P}<0.01-0.001)$. These time intervals must represent the mechanical delay of the muscle plus the latency for development of bone deformation (strain).

The mechanical effects of the MA and ZM on the SQ and CD strains are obvious, but the effect of the LP is not. Our previous data (18) indicated that isolated LP contraction, especially bilateral contraction, produces substantial strain on the CD but only trivial strain on the SQ. Thus, we speculated that the source of this CD strain was the direct load applied by the attachment and protrusive action of the LP on the CD (18). However, the present results do not support this direct mechanical effect because the lags for LP activity with CD and SQ strains were significantly longer than those of jaw adductor-strain pairs (145$152 \mathrm{~ms}$ versus $75-77 \mathrm{~ms}, \mathrm{P}<0.05-0.01)$. Therefore, the MA (probably including the ZM) is a more important source of condylar and squamosal loading than the LP. The observation that bone strain generally follows MA and ZM, but not LP activity, further strengthens this conclusion.

During the power stroke of mastication, the mandible is bent and twisted from MA contraction and bite force (27). The similar cross-correlation results from the MA/ZM-SQ/CD pairs and the MA-MD pair support this direct mechanical relationship between MA contraction and the development of MD strain. The inconsistent order between MA activity and MD strain, on the contrary, suggests that the MA is not the sole source of bone strain on the mandibular body. The additional source, however, is not the LP, as indicated by relatively long LP-MD lags with inconsistent signs. 


\section{Jaw muscle contraction and capsular deformation}

Unlike the bone strains that are the direct mechanical consequence of muscle contraction, capsular deformation of the jaw joint is probably mainly caused by jaw movement. The present results give further evidence that bone strains and capsular deformation are only indirectly related. First, the values of the coefficients of muscle-DVRT pairs were relatively low (approximately 0.50 , Table 3) regardless of which muscle was involved. Secondly, the lags were almost twice as long than those of muscle-strain pairs ( $130 \mathrm{~ms}$ versus $75 \mathrm{~ms}, \mathrm{P}<0.05)$. Thirdly, opposite to the sequence of jaw adductors with the SQ and CD strains, capsular deformation generally preceded MA activity (Table 3). Combined, these findings suggest that MA contraction did not cause capsular deformation.

Because the LP is associated with movements, a close relationship was expected between this muscle and capsular deformation. Specifically, the capsule shortens during opening, protrusion and contralateral shift (25), all movements associated with LP contraction. Only a tendency for LP activity to lead capsular deformation was seen $(P=0.11$, Table 3$)$, and the DVRT-LP pair did not show a shorter lag than the DVRT-MA pair. This failure to find the expected pattern may be due to the complicated firing pattern of the LP during mastication. Unlike the jaw adductors, LP can be active in a two-phase $(62.5 \%)$, a one-phase $(20.8 \%)$, or a mixed oneand two-phase $(16.7 \%)$ pattern (17) during mastication. Unfortunately, the current analysis was limited by the fact that LP activity was always defined as a single burst regardless of its pattern. This limitation made it difficult to detect order effects between LP activity and tissue deformation.

\section{Relationships among tissue deformations}

As discussed above, bone strain reflects applied load, whereas capsular deformation may relate to condylar movement. These events are not concurrent although muscle contractions cause both. This may explain why most DVRT-strain pairs showed relatively weak coupling ( 0.44 for average peak coefficient) and significant asynchrony (140 ms for average lag). The fact that capsular deformation occurred well prior to development of bone strain also shows these events are separate. The long lag (109 ms, Table 4) between left and right capsular deformations likely reflects the alternating lateral shift that occurs during mastication in pigs as discussed elsewhere $(17,25)$.
The results of bone strain comparison provide further evidence that $\mathrm{CD} / \mathrm{SQ}$ strains are related to each other but dissociated from MD strain. The MD strain always occurred later than SQ/CD strains (Table 4). As discussed above, $\mathrm{SQ} / \mathrm{CD}$ strains probably result directly from MA/ZM contraction. In contrast, MD strain must be related to a subsequent event, perhaps some aspect of occlusal contact.

\section{Coordination of jaw muscles}

The coefficient associated with each muscle pair provides an objective and systematic method for evaluating muscular coordination. In human mastication, the homologous and ipsilateral synergistic muscle pairs exhibit significantly greater coupling than do antagonistic or contralateral synergistic muscle pairs (8). However, the current results indicate that homologous and non-homologous muscle pairs did not differ in coupling strength, except for the increased lag of the MA-LP pair (Table 5). This apparent contradiction is probably artifactual. In Moore's report, a jaw closer (masseter muscle) was compared with an opener (anterior digastric muscle). In the present study, the "synergistic" MA and $\mathrm{ZM}$ are actually both parts of the masseter as defined in human anatomy (20). Furthermore, the LP is somewhat synergistic, instead of antagonistic, with the MA because both muscles contribute to contralateral shifts of the jaw. The longer lags in the non-homologous MA-LP pair may reflect the timing changes with each alternating chewing cycle in pigs.

\section{Conclusions}

In conclusion, the present study revealed that the time-course characteristics of jaw muscle contraction and tissue deformation showed regional and source specificity. As hypothesized, the jaw-adductor muscle activity was strongly coupled with the bone strains near the jaw joint. However, linkage of muscle activity and bone strain in the mandibular body was distinctively weak, indicating the existence of additional source of load. Coupling between the LP muscle and capsular deformation could not be confirmed, although this failure may be due to technical problems. Bone strain and capsular deformation of the jaw joint were not closely coupled, as expected. Homologous muscle pairs were nearly synchronous in their activities but their signals were not more similar to each other than those non-ho- 
mologous muscle pairs. The inherent relationships between muscle contraction and tissue deformations are intricate and outstrip their anatomic connections. Timeseries analysis is a helpful tool to unravel these complex interactions.

\section{Acknowledgments}

This project was supported by PHS grant 11962 from the National Institute of Dental and Craniofacial Research. We thank Katherine L. Rafferty, Christopher Marshall, and Scott C. Pedersen for their extensive help with the experiments, and Patricia Emry for general laboratory assistance.

\section{References}

1. Burr DB. Muscle strength, bone mass and age-related bone loss. J Bone Miner Res. 1997;12: 1547.

2. Herring SW, Liu ZJ. Loading of the TMJ: Anatomical and in vivo evidence from the bones. Cells Tissues Organs. 2001;169: 193.

3. Hylander WL, Johnson KR. The relationship between masseter force and masseter electromyogram during mastication in the monkey Macaca fascicularis. Arch Oral Biol. 1989;34: 713.

4. Hylander WL, Johnson KR. Modeling relative masseter force from surface electromyograms during mastication in non-human primates. Arch Oral Biol. 1993;38: 233.

5. Maier MA, Hepp-Reymond MC. EMG activation patterns during force production in precision grip. II. Muscular synergies in the spatial and temporal domain. Exp Brain Res. 1995;103: 123.

6. Oda S, Moritani T. Cross-correlation of bilateral differences in fatigue during sustained maximal voluntary contraction. Eur J Appl Physiol. 1995;70: 305.

7. Liu MM, Herzog W, Savelberg HHCM. Dynamic muscle force prediction from EMG: An artificial neural network approach. J Electromyogr Kinesiol. 1999;9: 391.

8. Moore CA. Symmetry of mandibular muscle activity as an index of coordinative strategy. J Speech Hear Res. 1993;36: 1145.

9. Schwartz AB, Adams JL. A method for detecting the time course of correlation between single-unit activity and EMG during a behavioral task. J Neurosci Methods. 1995;58: 127.

10. Gibbs J, Harrison LM, Stephens JA. Organization of inputs to motoneurone pools in man. J Physiol. 1995;485: 245.

11. Gibbs J, Harrison LM, Stephens JA. Cross-correlation analysis of motor unit activity recorded from two separated thumb muscles during development in man. J Physiol. 1997;499: 255.

12. Ruark JL, Moore CA. Coordination of lip muscle activity by 2 -year-old children during speech and nonspeech tasks. J Speech Hear Res. 1997;40: 1373.

13. Green JR, Moore CA, Ruark JL, Rodda PR, Morvée WT, van Witzenburg, MJ. Development of chewing in children from 12-48 months: Longitudinal study of EMG patterns. J Neurophysiol. 1997;77: 2704.

14. Green JR. Physiologic Development of Speech Motor Control: Articulatory Coordination of Lips and Jaw (Dissertation). Seattle, WA, USA: University of Washington; 1998.

15. Li L, Caldwell GE. Coefficients of cross correlation and the time domain correspondence. J Electromyogr Kinesiol. 1999;9: 385.

16. Semmler JG, Nordstrom MA. A comparison of cross-correlation and surface EMG techniques used to quantify motor unit synchronization in humans. J Neurosci Methods. 1999;90: 47.

17. Liu ZJ, Herring SW. Masticatory strains on osseous and ligamentous components of the jaw joint in miniature pigs. J Orofac Pain. 2000;14: 265.

18. Liu ZJ, Herring SW. Bone surface strains and internal bony pressures at the jaw joint during masticatory muscle contraction. Arch Oral Biol. 2000;45: 95.

19. Herring SW, Rafferty LK, Liu ZJ, Marshall CD. Jaw muscles and the skull in mammals: The biomechanics of mastication. J Comp Biochem Physiol. 2001;131: 207.

20. Herring SW. Physiology of feeding in miniature pigs. J Morphol. 1973;141: 427.

21. Biewener AA. In vivo measurement of bone strain and tendon force. In: Biewener AA, eds. Biomechanics - Structure and System. Oxford: Oxford Univ. Press; 1992: 123.

22. Beynnon BD, Fleming BC. Anterior cruciate ligament strain in vivo: A review of previous work. J Biomech. 1998;31: 519.

23. Herring SW. Mastication and maturity: A longitudinal study in pigs. J Dent Res. 1977;56: 1377.

24. Measurement Group. Strain gauge rosette-Selection, application and data reduction. Tech Note. 1990;515: 3.

25. Sun Z, Liu ZJ, Herring SW. Movement of TMJ tissues during mastication and passive manipulation: A study in miniature pigs. Arch Oral Biol. 2002;47: 293.

26. Rafferty KL, Herring SW, Artese F. Three-dimensional loading and growth of the zygomatic arch. J Exp Biol. 2000;203: 2093.

27. Hylander WL. Mandibular function in Galago crassicaudatus and Macaca fascicularis: An in vivo approach to stress analysis of the mandible. J Morphol. 1979;159: 253. 